\title{
Surface phonon polaritonics made simple: great as plasmonics but lower losses
}

\author{
Jamie M. Fitzgerald ${ }^{\mathrm{a}}$ and Vincenzo Giannini $\mathrm{i}^{\mathrm{b}, \mathrm{k}}$ \\ ${ }^{a}$ Chalmers University of Technology, Gothenburg, Sweden \\ 'Instituto de Estructura de la Materia, Consejo Superior de Investigaciones Científicas, Madrid, Spain
}

The manipulation of light and, in particular, the ability to guide it in an extremely sub-diffraction fashion, is a long-standing goal in nanophotonics. This capability will open up a plethora of applications and can be extremely useful for enhancing light-matter interactions. Such goals are historically associated with metals near their plasma frequency; this is known as plasmonics. ${ }^{1}$

The main limitation of plasmonics is the associated large losses of metals in the visible and near-IR, which prevents sharp resonances and a long propagation length of the surface waves. With time, researchers have started to explore new materials and different frequency regimes; similar physics was found in the mid-IR to THz region using "polar dielectrics" via the excitation of surface phonon polaritons (SPhPs). ${ }^{2}$ These are surface waves associated with the coupling of incident light with polar optical phonons and are supported within the materials' reststrahlen band. ${ }^{3}$ The mid-IR to THz region represents an exciting window for applications; it coincides with the IR-active vibrational fingerprints of a number of molecular species, which could be useful for trace chemical sensing. SPhPs have a significant advantage of low losses: the scattering time of optical phonons is on the order of $1 \mathrm{ps}$, in comparison to a typical 10 - to 100 -fs electron scattering time in metals. This, together with extreme light confinement far below the diffraction limit, leads consequentially to very high electromagnetic enhancement and long propagation lengths. ${ }^{3}$

Of particular interest is the fact that SPhPs occur in the same frequency region where graphene supports plasmons, representing a natural strategy to further shrink possible device dimensions. ${ }^{4}$ Despite the exceptional properties of SPhPs, they have a quite important practical drawback. It is not simple to manipulate phononic materials to obtain nanostructures. Many modern nanostructuring techniques can cause damage to the crystal lattice and lead to the incorporation of foreign ions on and near the surface, both of which lead to a significant reduction in the SPhP quality factor. This has somewhat limited the uptake of SPhPs in the nanophotonics community.

In this issue of Advanced Photonics, Qiang et al. ${ }^{5}$ are proposing a simple solution to this problem. They introduce a deep-subwavelength layer dielectric grating (70-nm-thick germanium), which is simpler to manufacture, on top of a flat phononic substrate ( $\mathrm{SiC}$ in this case). The grating can induce the excitation of localized SPhPs related to its groves as well as conveniently provide the necessary phase matching conditions for the excitation of propagating SPhPs. This has allowed the authors to study the interaction of localized and propagating phonon-polariton modes in a highly tunable system. By changing the grating width and period, the mixing of localized and propagating modes can be manipulated to provide adjustable resonant wavelength and field confinement of the hybrid modes in a desired spectral range. In this way, the authors show much-improved control of the nanophotonic properties of phononic materials. They are able to boast $Q$-factors

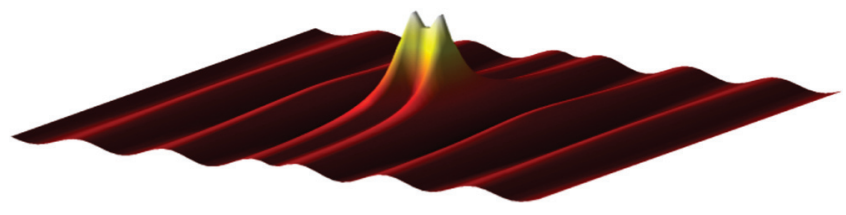

Artistic view of a phononic field.

of 96 and 195 for a localized dipolar resonance and a higher-order mode, respectively.

This work is an important step toward achieving the large theoretical predictions for the maximum quality factor of $\operatorname{SiC}^{3}(Q \sim 900)$ in a compact and on-chip platform. In particular, it could boost applications in quantum plasmonics. ${ }^{6}$ The indirect "structuring" of a material via the interaction with the near-field of a nearby nanofeature is proving to be a fruitful idea in nanophotonics. ${ }^{4}$ Such hybrid structures may well prove to be the next big thing in practical nanophotonic and nanophononic devices of the future.

\section{References}

1. V. Giannini et al., "Plasmonic nanoantennas: fundamentals and their use in controlling the radiative properties of nanoemitters," Chem. Rev. 111(6), 3888-3912 (2011).

2. A. J. Huber et al., "Focusing of surface phonon polaritons," Appl. Phys. Lett. 92, 203104 (2008).

3. J. D. Caldwell et al., "Low-loss, infrared and terahertz nanophotonics using surface phonon polaritons," Nanophotonics 4(1), 44-68 (2015).

4. K. Li et al., "Graphene plasmon cavities made with silicon carbide," ACS Omega 2(7), 3640-3646 (2017).

5. B. Qiang et al., "High $Q$-factor controllable phononic modes in hybrid phononic-dielectrics," Adv. Photonics 1(2), 026001 (2019).

6. J. M. Fitzgerald et al., "Quantum plasmonics," Proc. IEEE 104(12), 2307-2322 (2016).

Jamie M. Fitzgerald completed his $\mathrm{PhD}$ working on the topics of quantum plasmonics as well as surface phonon polaritons at Imperial College London. He is now a postdoctoral researcher at Chalmers University of Technology working on cavity optomechanics.

Vincenzo Giannini studied physics at the University of Pisa (Italy). He achieved his $\mathrm{PhD}$ in theoretical condensed matter physics at the CSIC in Madrid. He then had a postdoctoral position at the AMOLF, Amsterdam. Following, he enjoyed a Marie Curie fellowship at the Imperial College London. In June 2014, he started his group at the Imperial College London and recently he accepted an offer in Spain at the CSIC. 